\title{
Moon formation and orbital evolution in extrasolar planetary systems - A literature review
}

\author{
K. Lewis ${ }^{1}$ \\ ${ }^{1}$ Monash University, Clayton 3800, Australia [karen.lewis@sci.monash.edu.au]
}

\begin{abstract}
With over 450 extrasolar planets detected, the possibility of searching for moons of these planets is starting to be investigated. In order to make efficient use of limited observing resources, it would be useful if the types of moons that a given planet is likely to host was known prior to detection. Fortunately, informed by simulations of moon formation in our own solar system, as well as more general theoretical investigations of moon orbital evolution, such information is now available. I present a review of literature results concerning the likely physical and orbital properties of extra-solar moons, and how these properties are predicted to vary with the properties of their host planet.
\end{abstract}

\section{Introduction}

Before deciding which, if any, transiting planets should be investigated for moons, the following question needs to be considered: "What mass, radius and orbital parameters are the largest moons of a given planet likely to have?". This question can be addressed by considering the processes by which moons form, and their orbits subsequently evolve. Thus, informed by a review of large moons in our Solar System, the constraints resulting from moon formation and orbital evolution will be discussed in turn and collated into a summary of likely moon properties.

\section{Characteristics of Moons in the Solar System}

We begin our discussion of likely moon properties by summarizing the properties of moons in the Solar System. In particular, as a result of their different composition and formation history, the moons of terrestrial planets and gas giants will be discussed separately. We begin with a discussion of the moons of terrestrial planets.

\subsection{Moons of Terrestrial Planets}

Of the four terrestrial planets in our Solar System, only the Earth hosts a relatively large moon. Focussing our attention on the Earth-Moon system, it can be seen that it has a number of properties (see figure 1). It contains a single, large, moon which has a fair percentage $(\sim 1 \%)$ of the mass in the planet-moon system. In addition, the orbit of the Moon about the Earth is relatively circular and only slightly inclined $\left(\sim 5^{\circ}\right)$ with respect to the orbit of the Earth about the Sun. It is believed that the Earth-Moon system resulted from a giant impact, a process which will be discussed in section 3.1. 
EPJ Web of Conferences

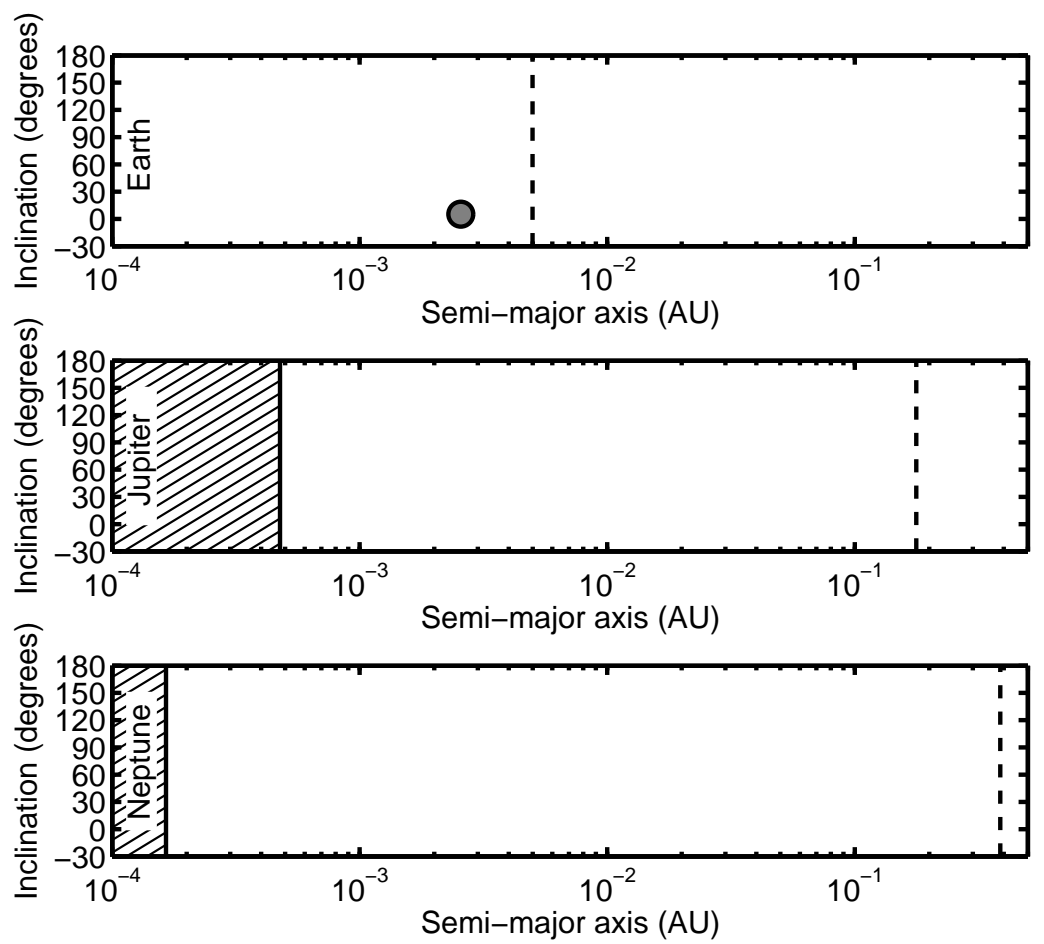

Figure 1: Schematics of the satellite systems of the Earth, Jupiter and Neptune. The regular satellites are shown as large filled dark grey circles with radii proportional to the radius of the corresponding satellite. Satellites too small to have a spherical shape are shown as small dots. The interior of the planet is represented by a hatched region on the left, while on the right a dashed line denotes half a planetary Hill radius.

\subsection{Moons of Gas Giant Planets}

Unlike the terrestrial planets, each of the four gas giant planets in our Solar System has a sequence of 1 to 7 large moons, along with a set of smaller moons. Focussing on these large moons as they are the most detectable, we begin by noticing that they all share a number of features, with one main exception, Triton, Neptune's only large moon. Consequently, the satellites of Jupiter, Saturn and Uranus will first be discussed, followed by a separate discussion of the properties of Triton.

The regular satellites of Jupiter (see figure 1), Saturn and Uranus share a number of features. Each planet has multiple large moons, each with mass comparable to that of the Moon, but small with respect to their host planet. In particular, the percentage of the planet-moon system's mass that is in regular satellites ranges from $0.025 \%$ to $0.011 \%$. Finally, the orbits of these moons are close to their host planet, circular and aligned with their planet's equator. A number of processes proposed which will produce such systems will be explained in section 3.2.

Compared to the satellite systems of the other gas giants, Neptune's satellite system is odd (see figure 1). First, it only has one large moon, Triton, and second, this 
moon's orbit is inclined and retrograde. However, similar to the moons of Jupiter, Saturn and Uranus, Triton is also relatively close to its host planet and not very massive compared to it. As a result of these properties it has been proposed that it was captured. This process will be discussed in section 3.3.

\section{Moon Formation Models}

The method by which a moon forms places limits on its physical and initial orbital properties. Within this context, moon formation resulting from a giant impact, a circumplanetary disk, and moon capture, will be discussed in turn.

\subsection{Terrestrial Planet: Impact Generated Moons}

Simulations of the period of chaotic growth, shows that giant impacts between planetary embryos, that are capable of forming moons, are common (e.g. Agnor, Canup, \& Levison 1999). Simulations of the impact process indicate that impacts between terrestrial mass proto-planets $\left(M_{p}<2.5 M_{\oplus}\right)$ produce a disk of orbiting debris (Wada, Kokubo, \& Makino 2006) which can coalesce into a moon a couple of planetary radii from its host. For standard sized terrestrial planets $\left(0.5-1 M_{\oplus}\right)$, the dynamics of the impact and interactions between the debris during post impact evolution generally result in a single (Canup \& Levison 1999), large moon, containing up to $4 \%$ of the planet's mass (e.g Canup, Ward, \& Cameron 2001). As a result of the random nature of the impact, this moon can have any initial orbital inclination. However, depending on this inclination, the dynamical evolution of the planet-moon system can result in re-impact, a moon on a close, inclined orbit, or a moon on a distant coplanar orbit (Atobe \& Ida 2007).

\subsection{Gas Giant: Disk Generated Moons}

It is believed that the regular satellites of Jupiter, Saturn and Uranus formed within a circumplanetary disk, as this explains the circular orbits of the regular satellites and their low inclination with respect to planetary rotation. In the literature, there are two main models describing the method by which a disk of gas and solids is processed into a small number of large satellites, that of Canup \& Ward (2006) and Mosqueira \& Estrada (2003a,b). Canup \& Ward (2006) use a time-dependant, single component circumplanetary disk model to investigate regular satellite formation. They suggest that the properties, in particular, the mass of regular satellites within this disk is determined by the balance between the rate of accretion of material onto the proto-moons, and orbital decay of these protomoons within the accretion disk onto the growing gas giant. This process results in a set of approximately 4 large moons within 60 planetary radii of the planet, with total mass approximately one ten thousandth of their host planet. In comparison, Mosqueira \& Estrada (2003a,b) propose a two-component disk model with a dense inner sub-disk extending out to the centrifugal radius, surrounded by a less dense outer disk. Unlike the model of Canup \& Ward (2006), this model predicts that the migration timescale of moons is much longer than their formation timescale. While the model does not provide a firm limit on moon mass, it does predict that at most one large satellite should form outside the centrifugal radius.

\subsection{Captured Satellites}

Planets can also capture large satellites. This occurs if a prospective moon suffers a close approach to a planet, looses sufficient energy through some process, e.g., threebody interaction (Agnor \& Hamilton 2006) or tidal dissipation (Podsiadlowski et al. 
2010), and ends up on a bound orbit. Immediately following capture, the moon is likely to be on a retrograde, highly eccentric orbit about its host planet. As the orbit of this new moon will cross the region in which regular satellites (if any) are likely to have formed, any existing large moons are likely to destroy, or be destroyed by the new moon. Following capture, the eccentricity of the moon's orbit tidally decays, resulting in a moon on a circular, retrograde orbit, with semi-major axis given by twice the initial periastron distance.

\section{Moon Orbital Evolution and Stability}

After formation, a moon's orbit may evolve, due to, e.g., tidal perturbations. This evolution will be discussed by first considering a planet and moon in isolation, second considering the effect of a star on this interaction, and finally, applying this understanding to the cases of moons of gas giants and terrestrial planets, and captured moons, in turn.

\subsection{Tidal Interaction Between a Planet and a Moon}

A moon's orbit is capable of exchanging angular momentum and energy with the rotation of the planet. In particular, this exchange and the associated orbital evolution can be thought of in terms of $\Omega_{p}$, the angular velocity associated with the rotation of the planet and $n_{m}$, the average angular velocity associated with the orbit of the moon. While the evolution process may be non-trivial (e.g. Hut 1980), there are only three possible outcomes corresponding to three possible initial conditions:

- Case 1: $\Omega_{p}>n_{m}$. In this case, energy and angular momentum are transferred to the moon's orbit, causing it to expand until synchronous rotation (the moon's orbit is circular, aligned with the planet's equator, and $n_{m}=\Omega_{p}$ ) is achieved. In particular, for low mass moons, the final size of the moon's orbit may be large.

- Case 2: $\Omega_{p}<n_{m}$ and the moon has "sufficient" angular momentum. For this case energy and angular momentum are transferred from the moon's orbit, to the planet's rotation causing the size of the moon's orbit to shrink until synchronous rotation is achieved.

- Case 3: $\Omega_{p}<n_{m}$, but in this case, the moon does not have enough angular momentum to transfer to the planet (as it is either too close or not massive enough). As a result, the moon will spiral into the planet.

\subsection{Affect of a Star on the Tidal Interaction Between a Planet and a Moon}

A star modifies the behavior described above in two ways. First, the tidal field of the star can modify a planet's rotational period such that, for the case where no other torques are applied to the planet, its rotational period evolves until it is equal to its orbital period, that is, it will become tidally locked. For the case of a planet tidally locked to its star, the moon semi-major axis corresponding to co-rotation is outside the Hill sphere of the planet, thus the orbits of all moons will evolve inwards and eventually spiral into their host planet. Second, if the orbit corresponding to the co-rotation radius is not three-body stable ${ }^{1}$, moons will be lost as a result of outward evolution before

\footnotetext{
${ }^{1}$ The set of stable moon orbits can be analytically estimated (e.g. Mardling 2008).
} 
they reach co-rotation. This is of particular importance if the moon is not very massive or the Hill radius is small. These two behaviors will be discussed for the case of moons of gas giants and terrestrial planets, and finally for captured moons.

\section{Orbital Evolution of Moons of Gas Giant Planets}

For the case of gas giant planets, a moon's orbits evolve in two different ways depending on the planet's semi-major axis. For close-in planets, moon lifetime was limited by inward orbital evolution and subsequent loss through impact with the planet, and for more distant planets, was limited by outward orbital evolution and eventual loss due to three body instability (Barnes \& O'Brien 2006). As moons of gas giant planets are expected to form close to their host planet (see section 3.2), moons of close-in gas giants are likely to be rapidly lost, while moons of distant gas giants may still be extant (see Weidner \& Horne 2010 for moon mass limits). This behavior depends on a range of factors, in particular, the $Q$-value of the planet, such that if a high value $\left(10^{12}\right)$ were used, large moons of hot Jupiters could be retained (Cassidy et al. 2009).

\section{Orbital Evolution of Moons of Terrestrial Planets}

For the case of moons of terrestrial planets, a similar analysis can be applied. Making the simplifying assumption that the planet's rotation is prograde, we again find that there are two regions of behavior. For close-in planets, impact generated moons are quickly lost as a result of inward migration. Alternatively, for more distant planets, the orbit of the moon can evolve outward and the moon retained. Consequently, we expect large impact generated moons to survive around more distant terrestrial planets, although we note that the planetary obliquity and moon orbital inclination can affect this result (Atobe \& Ida 2007).

\begin{tabular}{|c|c|c|c|}
\hline Property & $\begin{array}{c}\text { Moons of } \\
\text { Terrestrial Planets }\end{array}$ & $\begin{array}{c}\text { Moons of } \\
\text { Gas Giants }\end{array}$ & $\begin{array}{c}\text { Captured } \\
\text { Moons }\end{array}$ \\
\hline Multiplicity & Single & Multiple & Single \\
\hline Moon mass & $M_{m}<0.04 M_{p}$ & $M_{m}<2.5 \times 10^{-4} M_{p}$ & $\begin{array}{l}\text { Depends on method } \\
\text { of formation }\end{array}$ \\
\hline $\begin{array}{c}\text { Orbit } \\
\text { orientation }\end{array}$ & $\begin{array}{l}\text { Aligned with } \\
\text { planet orbit }\end{array}$ & $\begin{array}{l}\text { Aligned with } \\
\text { planet spin }\end{array}$ & $\begin{array}{l}\text { Retrograde w.r.t. } \\
\text { planet orbit }\end{array}$ \\
\hline $\begin{array}{l}\text { Semi-major } \\
\text { axis }\end{array}$ & $\begin{array}{l}\text { Determined by } \\
\text { orbital evolution }\end{array}$ & $\begin{array}{l}\text { Determined by } \\
\text { orbital evolution }\end{array}$ & Small \\
\hline
\end{tabular}

\section{Orbital Evolution of Captured Moons}

For the case of captured moons, the moon is likely to be in a retrograde orbit about its host. Again assuming that the rotation of the planet is prograde, the moon's orbit will gradually shrink until it impacts the planet. While captured moons have the disadvantage of having orbits that are likely to evolve inward, they may be large. In particular, the method of Podsiadlowski et.al. (2010) can produce gas giant-gas giant binaries, which should be easy to spot in transit light curves. 


\section{Conclusions}

Through an investigation of models of moon formation, and processes by which their orbits evolve, limits can be placed on the likely physical and orbital properties of moons of extra-solar planets (see table 1 ).

\section{References}

Agnor, C. B., Canup, R. M., \& Levison, H. F. 1999, Icarus, 142, 219

Agnor, C. B., \& Hamilton, D. P. 2006, Nature, 441, 192

Atobe, K., \& Ida, S. 2007, Icarus, 188, 1

Barnes, J. W., \& O'Brien, D. P. 2002, AJ, 572, 1087

Canup, R. M., \& Levison, H. F. 1999, ApJ, 117, 603

Canup, R. M., \& Ward, W. R. 2006, Nature, 441, 834

Canup, R. M., Ward, W. R., \& Cameron, A. G. W. 2001, Icarus, 150, 288

Cassidy, T. A., Mendez, R., Arras, P., Johnson, R. E., \& Skrutskie, M. F. 2009, ApJ, 704, 1341 Hut, P. 1999, A\&A, 92, 167

Mardling, R. M. 2008, in IAU Symp. 246, Dynamical Evolution of Dense Stellar Systems, ed. E. Vesperini, M. Giersz \& A. Sills (Cambridge: Cambridge University Press), 199

Mosqueira, I., \& Estrada, P. R. 2003a, Icarus, 163, 198

Mosqueira, I., \& Estrada, P. R. 2003b, Icarus, 163, 232

Podsiadlowski, P., Rappaport, S., Fregeau, J. M., \& Mardling, R. A. 2010, arXiv:1007.1418

Wada, K., Kokubo, E., \& Makino, H. 2006, ApJ, 638, 1180

Weidner, C., \& Horne, K. 2010, A\&A, 521, in press 